\title{
THE TWO-THIRDS RULE FOR DYNAMIC STORAGE ALLOCATION UNDER EQUILIBRIUM
}

\author{
Erol GELENBE* \\ Computer, Information and Control Engineering, University of Michigar, Ann Arbor, Michigan, USA
}

Received 25 February $19 ? 1$

dynamic storage allocation

fifty percent rule

equilibrum behavio:

\section{The two-thirds rule for dynamic storage allocation under equilibrium}

In this note we consider a dynamically allocated storage system for variable-size records, as described by Knuth [1] (Section 2.5). We assume that storage is allocated according to a "best-fit" strategy. Frea memory blocks are kept in the inked list AVAIL while USE is the set of reserved. blocks. Let $N$ and $M$ be the average number of blocks in USE and AVALL, respectively. As in [1] we assune that the system is in "equilibrium", sc that $N$ and $M$ remain constant. Let $(1-p)$ be the probability that a request for a block of size $x$ is satisfied exactly by one of the blocks in AVAIL. Knuth shows that under 'quilibrium implying that all requests of storage are satisfied (i.e. no overflow occurs),

$$
M=\frac{1}{2} p N
$$

and reports a simulation experiment verifying this relation when $p \approx 1$. Since usually $p \approx 1,(1)$ is known as the "fifty-percent rule" $[1,2]$.

The purpose of this communication is to derive a relationship hetween $f$, the average size $c:$ the blocks in AVAIL, and $r$ the average blcck size in USE. With this information one can then determine the fraction of sturage space which is reserved when th system operates under cquilitrium

\footnotetext{
* Systems Engineering Laboraiory, 2523 East Engineering. Ann Arbor, Michigan 48104.
}

$$
\rho=\frac{r N}{f M+r N}
$$

(i.e., $N, M$ are constant). It will be shown that for $p=1$

$$
\rho=\frac{2}{3},
$$

indicating that operation under equilibrium is inefficient in memory utilization. This value of $\rho$ is supported by Knuth's simulation experiment [1] since he reports that a memory overflow occurred (equilibrium was destroyed) if the reserved portion of the total memory capacity exceeded $\frac{2}{3}$.

The blocks in USE will be of three types, according to their location in memory with respect to the blocks in IVAIL. assuming that the memory locations are numbered consecutively $0,1, \ldots, K-1$ where $K$ is the capacity of the memory. Each block of the first type, $\alpha$, will have a free block at its "right" and "left"*. Each block of typ. $\beta$ will have a free block either at its right or left, but not both. Blocks of type $\gamma$ will have a block from USE at their right and left. There are $A, B, C$ blocks of type $\alpha, \beta, \gamma$ respectively in USE. Obviously [1]

$$
N=A+B+C
$$

\footnotetext{
* A block of memory is a set of consecutiveiy numbered memory lotations. If $\boldsymbol{n}$ is the largest location number a block occupier, then the block at its right begins at location $n+1$. The b'? ck at its left is defined analogously.
} 
Ad I pproximately (neglecting blocks at "edges" of tho memory)

$$
M=\frac{1}{2}(A+B)
$$

Let an event be an allocation of a block of free storaop to a record. Sincs the system is under equilibrium, Wh may assume that on the average only one block in USE enters AVAIL (becomes "free") in the time bctween two consecutive events. If $f$ is the average (over AVAIL) of free block sizes, after a block in USE enters AVAIL, $f$ shanges to

$$
\begin{aligned}
& f=\left[\frac{(M-2) f+2 f+r}{M-1}\right] \frac{A}{N} \\
& \quad+\left[\frac{(M-1) f+f+r}{M}\right] \frac{B}{N}+\left[\frac{\left.M \frac{j+r}{M}\right] \frac{C}{N}}{M+1}\right.
\end{aligned}
$$

since the newly frced block could have originated from $\alpha, \beta, \gamma$, witi probability $(A / N),(B / N),(C / N)$ reapectity ly*, and because if it is of type $\alpha$ the numbit of blocks in AVAIL will have decressed $M$ by one, white if it was of type $\gamma$ it would have increased $M$ by one. A newly freed block of type $\beta$ wonld leave $M$ unchanged. Therefoie

$$
\begin{aligned}
f=f & +\left[\frac{A}{N(M-1)}+\frac{B}{N M}+\frac{C}{N(M+1)}\right] \\
& +\left[\frac{A}{N(M-1)}-\frac{C}{N(M+1)}\right]
\end{aligned}
$$

eppronimately (for large $M$ )

$$
f=f+\frac{r}{M}+\frac{(A-C)}{M N} f
$$

Fouth [1] shows that unitr equilibrium

$$
C-A=(1-p) W,
$$

Q the owet type and lts lifotime are independont of each anter. therefore

$$
f^{\prime}=f+\frac{r}{M}-\frac{(1-F)}{M} f
$$

Immediately after an event, however. $r$ is changed to $f^{\prime \prime}$ as follows.

$$
f^{\prime \prime}=\left[\frac{(M-1) f^{\prime}+\left(f^{\prime}-r\right)}{M}\right] p+f^{\prime}(1-p)
$$

This is because if a perfect fit [with probability $(1-p)$ ] is obtained $M$ decreases by one and the average block in AVAIL remains of the same size, while if the fit is not perfect $M$ remains unchanged : ad a new block of size $\left(f^{\prime}-r\right)$ is added to AVAll. Thei ofore

$$
\begin{aligned}
f^{\prime \prime} & =f^{\prime}-\frac{p}{M} \\
& =f+\frac{r}{M}-\frac{(1-p)}{M} f-\frac{r p}{i l} .
\end{aligned}
$$

But if $f$ remains unchanged (in equilibrium) after each event we must have

$$
\frac{r}{M}-\frac{(1-p)}{M} f-\frac{r p}{M}=0
$$

herace

$$
r=f,
$$

independently of $p$. Thus from (2) we cotain

$$
\rho=\frac{1}{1+\frac{1}{2 p} p}
$$

vielding (3) when $p \approx i$.

\section{Refirences}

[1] D.E.Knuth, The Art of Computer Pros rananing, Volums 1, Fundamental Algorithms, deiwon-1/3y, Reding. Mass. 1968.

[2] P.J.Denning, Virtual Mlemor : Coanputing Surveys, VCl. 2, No. 3, September 1970,, $161-182$. 